\title{
Noninvasive Diagnostics in the Emergency Department: Just Because We Can Doesn't Mean We Should
}

We all want better, faster, cheaper, easier, and more convenient options for everything in our lives, and health care is no different. Theranos raised hundreds of millions of dollars on the false promise of performing laboratory analyses on microscopic amounts of blood because investors wanted to believe the impossible. ${ }^{1}$ The unrealized goals of avoiding painful blood draws (conveying patient satisfaction), reducing time delays associated with traditional clinical laboratories (conveying provider satisfaction), and improving sluggish patient throughput (conveying administrator satisfaction) keep us searching for expanded point-of-care testing opportunities in acute care settings. Well-established point-of-care testing modalities in the emergency department include finger-stick blood glucose measurement, 12-lead electrocardiograms, and bedside ultrasound. This issue of Respiratory CARE features 2 clinical studies examining minimally invasive and noninvasive diagnostic technologies and their usefulness for clinical screening of anemia ${ }^{2}$ and $\mathrm{CO}$ poisoning ${ }^{3}$ in the emergency department.

Context is important to determine how these studies may contribute to clinical care. In a seminal 1968 World Health Organization report, Wilson and Jungner base their list of fundamental criteria for screening on the statement that "the condition sought should be an important health problem." 4 Although both anemia and $\mathrm{CO}$ intoxication are unquestionably important medical conditions, the relative importance of detecting these etiologies depends heavily upon context. A general practitioner, a palliative care specialist, and an emergency physician would likely consider these pathologies with differing levels of concern. In the emergency department, the importance of either condition depends upon the interventions available for and the urgency of providing treatment, and testing is only important if treatment (or disposition) will change.

For a problem deemed important enough to merit screening efforts, any test used must be highly sensitive. A high

The authors have disclosed no conflicts of interest.

Correspondence: Jason T McMullan MD MS, Department of Emergency Medicine, University of Cincinnati, 231 Albert Sabin Way, Cincinnati, OH 45267. E-mail: jason.mcmullan@uc.edu.

DOI: $10.4187 /$ respcare. 07436 sensitivity ensures that a very large proportion of patients with the disease of interest are positively identified by the screening program and that few false negatives (missed patients) slip through the cracks. To minimize the number of false negatives, screening tests often sacrifice specificity at the expense of maximum sensitivity. In these cases, a second-tier confirmatory test is usually needed to rule out disease in the patients falsely testing positive. ${ }^{5}$

See the Original Studies on Pages 1343, 1351

Anemia complicates a wide array of medical conditions and is a risk factor for mortality in many other pathologies. ${ }^{6-8}$ The choice of treatment and the urgency of initiating therapy, however, varies significantly depending on the underlying cause. While incidentally discovered irondeficiency anemia will often improve with oral iron supplementation, hemorrhage requires source control and transfusion of blood products. The former condition is most likely to be managed on a non-emergent basis in the out-patient setting, whereas the latter mandates emergent resuscitation and urgent in-patient workup.

Citing potential usefulness for identifying occult anemia as the cause of nonspecific symptoms, Osborn and coauthors $^{2}$ compared the effectiveness of the noninvasive Masimo Pronto-7 photometric pulse CO-oximetry probe and the HemoCue $\mathrm{Hb} \mathrm{201+} \mathrm{capillary} \mathrm{blood} \mathrm{sampling} \mathrm{de-}$ vice against laboratory quantification of hemoglobin concentration in a convenience sample of subjects in the emergency department. Although the authors concluded that both technologies were sufficiently accurate for detecting anemia, many clinicians would not accept the $82 \%$ sensitivity reported for the Pronto-7 device. Although the $\mathrm{Hb} 201+$ device demonstrated a superior sensitivity of $99 \%$, the importance of screening for anemia in the emergency department by any method remains questionable. ${ }^{2}$ Unstable patients, who may most benefit from rapid assessment, were specifically excluded from this investigation. Patients with concerning histories or clinical exam findings are likely to have a complete blood count ordered, which provides substantially more diagnostic information than a simple hemoglobin level. For the remaining patients 


\section{EDITORIALS}

whose clinical presentations do not merit even basic laboratory workup, it is hard to imagine many have hemoglobin levels below the threshold for blood transfusion $(7 \mathrm{~g} / \mathrm{dL})$. Detecting anemia in these patients is therefore unlikely to impact their management in the emergency department.

CO toxicity, like occult anemia, may present with a wide range of nonspecific symptoms such as headache, nausea, or dizziness. Severe acute intoxication may lead to seizures, syncope, arrhythmia, or coma. Treatment with high-flow supplemental oxygen is the mainstay of care, although critically ill patients may require hyperbaric oxygen therapy to reduce the risk of delayed neurological sequelae and subsequent long-term cognitive impairment. ${ }^{9}$ After treating the patient, efforts can be undertaken to identify the source of $\mathrm{CO}$ poisoning and prevent additional exposure. For these reasons, rapid recognition of $\mathrm{CO}$ toxicity is desirable.

Unfortunately, CO toxicity is frequently overlooked or recognized only after a considerable delay. The low prevalence of the condition (estimated between $0.05 \%$ to $0.24 \%$ in the emergency department $)^{10,11}$ probably poses a barrier to its serious consideration in some of these cases. Routine blood gas analysis used to investigate acid/base status or respiratory status does not include measurement of $\mathrm{CO}$ levels; therefore, CO-oximetry is unlikely to be obtained unless $\mathrm{CO}$ poisoning is part of the clinician's differential diagnosis. While a patient presenting from the scene of a structure fire with a chief complaint of vomiting is likely to arouse suspicion for $\mathrm{CO}$ intoxication, many patients do not report a clear history of exposure. ${ }^{12}$ At present, definitive diagnosis still hinges upon detection of an elevated carboxyhemoglobin ( $\mathrm{HbCO}$ ) level by blood gas CO-oximetry. ${ }^{9}$

Because recognizing $\mathrm{CO}$ intoxication can be difficult, development of an efficient and cost-effective method of screening patients in the emergency department for elevated $\mathrm{HbCO}$ levels could provide a benefit to patient care by expediting initiation of oxygen therapy. Pulse CO-oximetry, a noninvasive test with rapidly available results, seems well suited for such an application. This test, however, indirectly measures $\mathrm{HbCO}$ using peripheral capillary $\mathrm{CO}$ saturation as a surrogate marker. Multiple studies over the last decade have compared the accuracy of pulse COoximetry against the accepted standard of blood gas analysis, yielding generally broad limits of agreement between the tests and an inconsistent range of sensitivities for detecting elevated $\mathrm{CO}$ levels using peripheral capillary $\mathrm{CO}$ saturation. ${ }^{13-16}$ Despite overcoming the limitations of previous investigations, the study by Villalba and coauthors in this issue of the Journal ${ }^{3}$ revealed wide limits of agreement between pulse CO-oximetry and blood gas analysis $(-10.3 \%$ and $8.1 \%)$. Using a commonly cited cutoff value of $10 \% \mathrm{HbCO}$ for significant CO toxicity, ${ }^{12,15,17}$ Villalba and coauthors further demonstrated that pulse CO-oximetry had a poor sensitivity of $60.1 \% .^{3}$

At present, the literature does not support independent use of pulse $\mathrm{CO}$-oximetry for determining $\mathrm{HbCO}$ levels because the false negative rate is unacceptably high. Even the manufacturer of the studied pulse CO-oximetry technology states that the devices are "not to be used as the sole basis for making diagnosis or treatment decisions" (https://www.masimo.com/products/continuous/radical-7, Accessed August 26, 2019). Unfortunately, $>40 \%$ of hospitals in some regions of the United States do not have the ability to obtain blood gas results in real time, and a 2-tiered screening regimen is not feasible in such areas. ${ }^{18,19}$ Foregoing confirmatory testing and empirically administering $100 \%$ oxygen to everyone with a positive screen, however, would certainly expose some individuals to an unnecessary risk of oxygen toxicity. Considering the low prevalence of $\mathrm{CO}$ intoxication, the substantial number of poisoned patients potentially missed due to the limited sensitivity of pulse CO-oximetry, and the need for confirmatory testing that is not universally available, a strong argument in favor of screening using peripheral capillary $\mathrm{CO}$ saturation levels does not exist.

Screening initiatives for anemia or CO poisoning using the minimally invasive and noninvasive devices described above are not currently justified, albeit for different reasons. A patient whose presentation does not merit laboratory workup in the emergency department is unlikely to receive any acute intervention for an incidentally discovered anemia. Conversely, early detection of $\mathrm{CO}$ toxicity in an undifferentiated patient could lead to more rapid initiation of oxygen therapy, but uncertainties surrounding the dependability of pulse CO-oximetry technology currently stand in the way of effective screening initiatives. When deciding to implement a screening program based in the emergency department, one must be able to offer a meaningful intervention upon detection of a true positive result. Likewise, one must be certain that a large proportion of afflicted patients do not escape detection. Point of care hemoglobin and $\mathrm{CO}$ assessment may indeed have useful applications in the emergency department setting, provided they are used to ask the right question. Based on the work by Osborn et $\mathrm{al}^{2}$ and Villalba et $\mathrm{al}^{3}{ }^{3}$ neither technology offers clinical utility for the screening purposes investigated.

\section{Andrew N Hogan} Jason T McMullan

Division of Emergency Medical Services Department of Emergency Medicine University of Cincinnati Cincinnati, Ohio 


\section{EDITORIALS}

\section{REFERENCES}

1. Carreyrou J. Hot startup Theranos has struggled with its blood-test technology. The Wall Street Journal: Oct 16, 2015. Available at: https://www.wsj.com/articles/theranos-has-struggled-with-bloodtests-1444881901. Accessed September 25, 2019.

2. Osborn ZT, Villalba N, Derickson P, Sewatsky TP, Wager A, Freeman K. Accuracy of point of care testing for anemia in the emergency department. Respir Care 2019;64(11):1343-1350.

3. Villalba N, Osborn ZT, Derickson P, Manning C, Herrington R, Kaminsky D, Freeman K. Diagnostic performance of carbon monoxide testing by pulse oximetry in the emergency department. Respir Care 2019;64(11):351-1357.

4. Wilson JMG, Jungner G. Principles and practice of screening for disease. Geneva: World Health Organization; 1968. Available at: https:// apps.who.int/iris/handle/10665/37650. Accessed August 25, 2019.

5. Herman C. What makes a screening exam "good"? Virtual Mentor 2006;8:34-37.

6. Foley RN, Parfrey PS, Harnett JD, Kent GM, Murray DC, Barre PE. The impact of anemia on cardiomyopathy, morbidity, and mortality in end-stage renal disease. Am J Kidney Dis 1996;28(1):53-61.

7. Caro JJ, Salas M, Ward A, Goss G. Anemia as an independent prognostic factor for survival in patients with cancer: a systemic, quantitative review. Cancer 2001;91(12):2214-2221.

8. He SW, Wang LX. The impact of anemia on the prognosis of chronic heart failure: a meta-analysis and systemic review. Congest Heart Fail 2009;15(3):123-130.

9. Ng PC, Long B, Koyfman A. Clinical chameleons: an emergency medicine focused review of carbon monoxide poisoning. Intern Emerg Med 2018;13(5):825.

10. Hampson NB. Emergency department visits for carbon monoxide poisoning in the Pacific Northwest. J Emerg Med 1998;16(5):695698.
11. Partrick M, Fiesseler F, Shih R, Riggs R, Hung O. Monthly variations in the diagnosis of carbon monoxide exposures in the emergency department. Undersea Hyperb Med 2009;36(3):161-167.

12. Hampson NB, Piantadosi CA, Thom SR, Weaver LK. Practice recommendations in the diagnosis, management, and prevention of carbon monoxide poisoning. Am J Respir Crit Care Med 2012;186(11): 1095-1101.

13. Touger M, Birnbaum A, Wang J, Chou K, Pearson D, Bijur P. Performance of the RAD-57 pulse co-oximeter compared with standard laboratory carboxyhemoglobin measurement. Ann Emerg Med 2010;56(4):382-388.

14. Roth D, Herkner H, Schreiber W, Hubmann N, Gamper G, Laggner AN, Havel C. Accuracy of noninvasive multiwave pulse oximetry compared with carboxyhemoglobin from blood gas analysis in unselected emergency department patients. Ann Emerg Med 2011; 58(1):74-79.

15. Sebbane M, Claret PG, Mercier G, Lefebvre S, Théry R, Dumont R, et al. Emergency department management of suspected carbon monoxide poisoning: role of pulse CO-oximetry. Respir Care 2013;58(10): 1614-1620.

16. Weaver LK, Churchill SK, Deru K, Cooney D. False positive rate of carbon monoxide saturation by pulse oximetry of emergency department patients. Respir Care 2013;58(2):232-240.

17. Chee KJ, Nilson D, Partridge R, Hughes A, Suner S, Sucov A, Jay G. Finding needles in a haystack: a case series of carbon monoxide poisoning detected using new technology in the emergency department. Clin Toxicol (Phila) 2008;46(5):461-469.

18. Hampson NB, Scott KL, Zmaeff JL. Carboxyhemoglobin measurement by hospitals: implications for the diagnosis of carbon monoxide poisoning. J Emerg Med 2006;31(1):13-16.

19. Masters T, Willenbring B, Westgard B, Cole J, Hendriksen S, Walter $\mathrm{J}$, et al. Availability of bedside and laboratory testing for carbon monoxide poisoning in the upper Midwestern United States. West J Emerg Med 2019;20(3):506-511. 